Ophthalmic artery blood flow

\section{Ophthalmic artery blood flow in humans}

\section{T R Hedges}

\section{The tortuosity and the variable course of the ophthalmic artery remain a problem}

E fforts to analyse the complex vasculature of the eye have been frustrating to say the least. We still must rely very much on fluorescein angiography. Laser Doppler flowmetry has not yet yielded the results that had been hoped for. The same has been true of colour Doppler imaging. In a recent attempt on my part to make sense of many techniques used to study the circulation of the optic nerve, my conclusion was that much work still needs to be done. ${ }^{1}$ Harris and colleagues are to be commended for relentlessly struggling to find better ways to determine how blood flow can change under a variety of clinical settings using several techniques, especially colour Doppler imaging.

Until recently, colour Doppler imaging has been limited to visualising blood vessels, identifying direction of blood flow, and calculating blood velocity only. Pulsatility and resistive indices provide indirect evidence of resistance at or nearby the ultrasound probe, but volumetric blood flow (amount of blood/ time) has not been measurable. The main problem with accurately assessing the orbital vessels has been the small size of the ophthalmic artery and especially the central retinal and ciliary arteries. In the paper by Orge et al in this issue of the $B J O$ (p 1216) the ophthalmic artery, because of its relatively larger size, was measured with regard to diameter in order to calculate volume and, ultimately, blood flow. However, it is evident that these investigators are still wrestling with considerable variability in measurements within their own laboratory.

The limitations of the authors' methods are reflected in their interobserver coefficients of variability of $40 \%$ and intraobserver variability of almost 30\% for calculations of blood flow. This wide range of measurements may simply have been due to the small size of the ophthalmic artery, but it may also be due to its tortuous course and the fact that it contains many branches. Among different individuals, ophthalmic arteries also have large variations in size and configuration, which will inherently make comparing blood flow between different patients subject to error. These issues require that the technicians performing each study employ a great deal of judgment, which in turn adds another layer of variability to any study, especially if this technique is to be applied to patients with disease.

With regard to size of the artery, previous efforts to measure blood flow, using similar methods employed by Ogre et al, have been made with veins, particularly shunts for renal dialysis. Even with this application, accuracy is limited. With respect to measuring arteries elsewhere in the body, there is evidence that large vessels may be accurately measured. However, the error in measuring smaller vessels is obvious. In one study, mean flow and standard errors in the common femoral artery was calculated to be 284 (SE 21 ) $\mathrm{ml} / \mathrm{min}$, whereas in the dorsalis pedis it was 3 (1) $\mathrm{ml} / \mathrm{min}^{2}$ As Orge et al point out, one is limited to measuring only a very few pixels on the screen. With regard to an artery as small as the ophthalmic artery, which is about $2 \mathrm{~mm}$ in diameter, one is basically counting about 10 pixels. If one considers that converting a diameter measurement to the measurement of area, the error would be squared. When we attempted to measure ophthalmic artery diameter in our own laboratory, we found that repeated measurements could easily be wrong by at least one or two pixels. We do not have the advantage of the modified software that Ogre et al refer to in their paper, but even when we employed a new technique called "B flow," our accuracy in measuring ophthalmic artery diameters was still, in our opinion, poor.

The ophthalmic artery is also quite tortuous. In some individuals a consistently measurable segment of the artery can be identified, but in many others only a very short segment can be seen at any one time. Although the authors state "there is no need for assumptions about turbulence of flow," "since each pixel within the image of moving blood contains a single velocity measurement," we still remain concerned that turbulence of blood flow within the ophthalmic artery may add another source of error especially with regard to velocity measurements. Furthermore, the ophthalmic artery contains many branches, which are also known to affect blood flow velocity.

A variety of other issues have been raised by others involved in attempting to measure blood flow velocity using Doppler ultrasound. These include nonuniform insonation of the blood vessel, differential attenuation between soft tissue and blood, intrinsic spectoral broadening, frequency dependent scattering, high pass filtering designed to reduce high amplitude, low frequency, Doppler shifts due to vessel wall motion, sheer rate and haematocrit, as well as poor signal to noise ratio.

Although Ogre et al show that blood flow within the ophthalmic artery can be estimated, variability, due to the small numbers generated from changes measured in small arteries, limits colour Doppler imaging to more qualitative rather than accurate quantitative analysis of ophthalmic artery blood flow. Even if the resolution of the method could be refined, the problem of the tortuosity and the variable course of the ophthalmic artery remains an issue. Finally, blood flow in any blood vessel is subject to systemic haemodynamics. Harris and his coworkers may have reached the point where blood flow can be measured in the ophthalmic artery to a limited degree. However, at this time, we should remain cautious regarding application of this method to clinical studies.

Br J Ophthalmol 2002;86:1197

\section{Author's affiliations}

T R Hedges, Tufts University School of Medicine, New England Medical Center, Boston, Massachusetts, USA

\section{REFERENCES}

1 Hedges TR. Assessing the vascular supply of the optic disc. Presented at the North American Neuro-ophthalmology Society Meeting, Rancho Mirage, California, 21 February 2001

2 Holland CK, Brown JM, Scoutt LM, et al. Lower extremity volumetric arterial blood flow in normal subjects. Ultrasound Med Biol 1998;24:1079-86. 


\section{The evolving management of optic nerve sheath meningiomas}

\section{N R Miller}

\section{Consider using stereotactic fractionated irradiation}

T he management of optic nerve sheath meningiomas (ONSMs) has

been a controversial and sometimes contentious issue for a number of reasons. In the first place, these tumours are not associated with any mortality or significant neurological morbidity. In the second place, although they theoretically can spread across the planum sphenoidale to affect the opposite optic nerve, they rarely do so and, thus, almost never cause loss of vision in the opposite eye. The major manifestation of ONSMs, therefore, is monocular visual loss, and their management should reflect these issues.

Because primary ONSMs arise from the arachnoid cap cells surrounding the intracanalicular or intraorbital portions of the nerve, they almost always are intimately associated with the nerve and tend to surround the nerve by the time they become clinically apparent. Similarly, secondary ONSMs extend from the planum sphenoidale into the subdural or subarachnoid spaces (or both) surrounding the nerve within the optic canal and, ultimately, within the orbit. As if that were not enough, many ONSMs involve the portion of the optic nerve at the apex of the orbit within the annulus of Zinn, an area not amenable to safe and comprehensive surgical exploration. Thus, despite a few reports indicating preservation of vision following removal of an ONSM, attempts to cure this tumour surgically almost always produce blindness in the affected eye-precisely what one is trying to avoid in the first place-as well as ophthalmoparesis from damage to ocular motor nerves and/or extraocular muscles. If, on the other hand, the optic nerve is removed along with the tumour, the patient may be "cured," but the procedure will, of course, cause blindness in an eye that may have had useful vision and that may have retained that level of visual function for several years without intervention and, in addition, there will be at least temporary ophthalmoparesis.

Although the potential for hormone therapy for meningiomas, the cells of which usually possess oestrogen and/or progesterone receptors, has received considerable attention, clinical studies using tamoxifen (an anti-oestrogen agent) and mefipristone (an antiprogesterone agent), have been disappointing. Thus, until recently, the most popular management options for a patient with a presumed ONSM have been observation without intervention and surgical excision of the tumour along with the optic nerve.

Earlier this year, Turbin et al ${ }^{1}$ provided substantial data indicating that conventional fractionated radiation therapy is a better treatment for ONSMs than either surgery or no intervention. Although other authors had reported similar results, ${ }^{23}$ the study by Turbin et al is the most comprehensive to date. Over a mean follow up period of 10 years, the patients in their study who received conventional fractionated radiation therapy alone had a substantially better visual outcome than did patients who were observed without intervention or patients who underwent surgery, and this was the case regardless of the level of vision when treatment was initiated. Thus, it is clear that radiation therapy should be considered for most patients with an ONSM

Unfortunately, radiation therapy for ONSMs is not without potential side effects. Although these are often mild and transient (for example, local erythema and local alopecia), the potential for severe visual loss from radiation induced retinopathy, optic neuropathy, or even damage to the optic chiasm and/or contralateral optic nerve cannot be ignored. Thus, in recommending radiation therapy to a patient with an ONSM, one must consider the potential side effects that could damage the vision that one is attempting to preserve or improve, and any method that could prevent or reduce the frequency and severity of these side effects would be welcome. In this issue of the BJO (p 1265), Pitz et al report the results of treatment of 15 patients with ONSMs using a more precise form of fractionated radiation therapystereotactic fractionated conformal irradiation. This technique should not be confused with stereotactic radiosurgery. The latter techinique consists of the delivery of a single, large dose of focused radiation (for example, 40-50 Gy) (or, occasionally, several large daily doses) to the lesion, whereas the technique described by the authors is basically the same as conventional fractionated radiation therapy (for example, 1.8-2 Gy per day to a total dose of about $50 \mathrm{~Gy}$ ), except that much more precise delivery of radiation is achieved through use of a three dimensional stereotactic system, thus reducing the extent of spread of radiation and, therefore, the potential for damage to surrounding structures, particularly the globe, the optic chiasm, and the contralateral optic nerve. In their study, Pitz et al found that over a mean follow up period of 37 months after completion of treatment, there was no evidence of tumour growth or extension, none of the treated seeing eyes experienced deterioration of vision, and several eyes improved with respect to visual acuity, visual field, or both. Perhaps, most importantly, none of the radiated eyes developed radiation retinopathy or optic neuropathy, and none of the contralateral eyes showed evidence of radiation injury. Although the follow up period may have been too short to observe spread of an inadequately treated tumour or to observe any major ocular complications of the radiation, the results of Pitz et al suggest that stereotactic fractionated radiation therapy for ONSM may produce a better long term visual result with fewer vision threatening side effects than conventional fractionated radiation therapy. If this is truly the case, one would have little reason to delay treatment of ONSMs in patients with good visual sensory function because of fear of radiation side effects or inadequate tumour treatment. It is thus to be hoped that the results reported by Pitz et al will stand up over the next 10-20 years of follow up. In the meantime, I would urge all physicians to consider using stereotactic (three dimensional conformal) fractionated irradiation rather than conventional fractionated irradiation for their patients with presumed ONSMs.

Br J Ophthalmol 2002;86:1198

\section{Author's affiliations}

N R Miller, Wilmer Eye Institute, Baltimore, MD 21287, USA; nrmiller@jhmi.edu

\section{REFERENCES}

1 Turbin RE, Thompson CR, Kennerdell JS, et al. A long-term visual outcome comparison in patients with optic nerve sheath meningioma managed with observation, surgery, radiotherapy, or surgery and radioatherapy. Ophthalmology 2002; 109:890-9.

2 Smith JL, Vuksanovic MM, Yates BM, et al. Radiation therapy for primary optic nerve meningiomas. J Clin Neuroophthalmol meningiomas.

3 Kennergell JS, Maroon JC, Malton M, et al. The management of optic nerve sheath meningiomas. Am J Ophthalmol 1988;106:450-7. 